\title{
The Critical Role of Infectious Disease in Compensatory Population Growth in Response to Culling
}

\author{
Eleanor Tanner, ${ }^{1, *}$ Andy White, ${ }^{1}$ Peter W. W. Lurz, ${ }^{2}$ Christian Gortázar, ${ }^{3}$ Iratxe Díez-Delgado, ${ }^{3}$ \\ and Mike Boots ${ }^{4}$
}

1. Maxwell Institute for Mathematical Sciences, Department of Mathematics, Heriot-Watt University, Edinburgh EH14 4AS, United Kingdom; 2. Lurzengasse 3, D-97236 Randersacker, Germany; 3. SaBio, Instituto de Investigación en Recursos Cinegéticos (IREC; Consejo Superior de Investigaciones Científicas [CSIC]/Universidad de Castilla-La Mancha [UCLM]), Ciudad Real, Spain; 4. Department of Integrative Biology, University of California, Berkeley, California 94720; and Biosciences, College of Life and Environmental Sciences, University of Exeter, Cornwall Campus, Treliever Road, Penryn, Cornwall TR10 9EZ, United Kingdom

Submitted September 14, 2017; Accepted December 24, 2018; Electronically published May 29, 2019

Online enhancements: appendix, Matlab code.

\begin{abstract}
AвsтRACT: Despite the ubiquity of disease in nature, the role that disease dynamics play in the compensatory growth response to harvesting has been ignored. We use a mathematical approach to show that harvesting can lead to compensatory growth due to a release from diseaseinduced mortality. Our findings imply that culling in systems that harbor virulent parasites can reduce disease prevalence and increase population density. Our models predict that this compensation occurs for a broad range of infectious disease characteristics unless the disease induces longlasting immunity in hosts. Our key insight is that a population can be regulated at a similar density by disease or at reduced prevalence by a combination of culling and disease. We illustrate our predictions with a system-specific model representing wild boar tuberculosis infection, parameterized for central Spain, and find significant compensation to culling. Given that few wildlife diseases are likely to induce long-lived immunity, populations with virulent diseases may often be resilient to harvesting.
\end{abstract}

Keywords: culling, disease dynamics, virulence, immunity, compensatory growth, hydra effect.

\section{Introduction}

It is well known that harvesting may be compensated by an increased growth rate at lower density (Abrams 2009). This phenomenon of compensatory growth in response to culling was first modeled by Ricker (1954), who showed that for moderate harvesting levels the population can stabilize to a

\footnotetext{
* Corresponding author; email: ent1@hw.ac.uk.

ORCIDs: Tanner, https://orcid.org/0000-0003-4375-725X; White, https:// orcid.org/0000-0002-0677-5531; Gortázar, https://orcid.org/0000-0003-0012 -4006; Díez-Delgado, https://orcid.org/0000-0002-8898-1960; Boots, https:// orcid.org/0000-0003-3763-6136.

Am. Nat. 2019. Vol. 194, pp. E1-E12. (C) 2019 by The University of Chicago. 0003-0147/2019/19401-57961\$15.00. All rights reserved.

DOI: $10.1086 / 703437$
}

level that exceeds the density in the absence of harvesting. Despite the ubiquity of infectious disease in nature, little work has considered the impact of harvesting and culling in populations with virulent infectious disease and the compensatory potential of changes to disease dynamics. Since culling affects the disease dynamics, there is considerable potential to generate feedbacks on host population dynamics. Moreover, as culling is also used as a management strategy to control emergent wildlife diseases, it is vital to understand the interplay among culling, disease, and population dynamics (Barlow 1996; Woodroffe 1999). In this study, we develop mathematical models to examine the impact of culling in systems that support endemic diseases and for the first time detail how culling can lead to compensatory growth due to a reduction in disease-induced mortality.

It is difficult to gather field data to test theories about the population-level implications of complex disease dynamics (Abrams 2009; McCallum 2016). Mathematical models are therefore important tools for explaining the impact of culling and harvesting in systems with endemic parasites. There is an extensive modeling literature focused on the control of disease through culling, for example, chronic wasting disease in deer in North America (Potapov et al. 2012; Storm et al. 2013; Uehlinger et al. 2016; Wasserberg et al. 2009), acutely virulent classical swine fever in wild boar (Choisy and Rohani 2006; Bolzoni et al. 2007; Cowled et al. 2012), and lethal facial tumor disease in Tasmanian devils (Beeton and McCallum 2011). While these studies recognize that culling in systems with endemic disease can induce compensation through demographic processes, they have not examined how culling may lead to compensatory effects that arise directly from changes in disease dynamics.

The limited work that has examined the effect of culling on disease dynamics has shown that culling may increase prev- 
alence due to a decrease in long-lasting immunity or vaccine coverage (Choisy and Rohani 2006; Bolzoni et al. 2007; Potapov et al. 2012), with Potapov et al. (2012) showing that prevalence can decrease in a system with no immunity. However, these studies have not examined compensatory effects due to disease and have only considered a limited range of disease characteristics. Here, we model in general the impacts of culling and harvesting in systems that support a wide range of endemic diseases. A novel aspect of our study is that we isolate the compensatory effects following culling due to changes in the disease dynamics resulting from a population-level release from disease-induced mortality. This facet is vital if we are to understand the response of harvesting in managed and natural systems that harbor virulent parasites. We show that significant host compensation occurs in response to culling for infections that do not cause long-lasting immunity, and therefore such host populations can be more sustainable. However, when there is long-lasting immunity the disease can decrease the population's resilience to harvesting and increase its extinction risk when culling is used to manage a disease. We develop a system-specific model of Sus scrofa (wild boar) tuberculosis (TB) interactions that illustrates our predictions in a specific disease context. Our work highlights the importance of understanding the nature of immunity to infectious disease for sustainable harvesting of populations and the management of disease through culling.

\section{Methods}

We examine a classical compartmental susceptible-infectedrecovered-susceptible (SIRS) model of disease (Anderson and May 1979; Keeling and Rohani 2008) that considers a total population $(N)$ split into separate classes representing different disease stages - the class of susceptibles $(S)$, of infecteds $(I)$, and of recovereds/immunes $(R)$ - such that the total population density is $N=S+I+R$. In this model, all classes reproduce, and all newborns are susceptible. The maximum per capita birth rate $b$ decreases with increasing density through parameter $q$, and all population classes incur natural death at rate $d$. A susceptible individual becomes infected with transmission rate function $\theta(I, N)$, which can represent densitydependent disease transmission (hereafter, DD transmission) or frequency-dependent disease transmission (hereafter, FD transmission; eqq. [4] and [5], respectively). Infected individuals incur additional disease-induced mortality (virulence) at rate $\alpha$ and can recover from infection to become immune from the disease at rate $\eta$. Immunity can be lost, causing recovereds to become susceptible again at rate $\eta$. This model is represented with the following system of ordinary differential equations:

$$
\frac{\mathrm{d} S}{\mathrm{~d} t}=b N(1-q N)-d S-\theta(I, N) S+\eta R,
$$

$$
\begin{aligned}
& \frac{\mathrm{d} I}{\mathrm{~d} t}=\theta(I, N) S-d I-\alpha I-\gamma I, \\
& \frac{\mathrm{d} R}{\mathrm{~d} t}=\gamma I-d R-\eta R .
\end{aligned}
$$

A strength of our model is that it can be adapted to represent a range of classical infection frameworks: SI by setting $\gamma=$ 0 , SIR by setting $\gamma>0$ and $\eta=0$, and SIRS by setting both $\gamma>0$ and $\eta>0$. The system is normalized to a common endemic steady state $N=N_{\mathrm{e}}\left(S=S_{\mathrm{e}}, I=I_{\mathrm{e}}\right.$, and $R=R_{\mathrm{e}}$, and we choose $N_{\mathrm{e}}=1$ without loss of generality) when the initial prevalence prior to culling is $p_{\mathrm{i}}\left(=I_{\mathrm{e}} / N_{\mathrm{e}}\right.$ ) (for further details, see sec. A1 of the appendix, available online). The endemic transmission functions are defined as follows:

$$
\begin{gathered}
\text { DD transmission: } \theta(I, N)=\frac{d+\alpha+\gamma}{N_{\mathrm{e}}\left(1-p_{\mathrm{i}}\left(\frac{\gamma}{d+\eta}+1\right)\right)} I, \\
\text { FD transmission: } \theta(I, N)=\frac{d+\alpha+\gamma}{\left(1-p_{\mathrm{i}}\left(\frac{\gamma}{d+\eta}+1\right)\right)} \frac{I}{N} .
\end{gathered}
$$

Under this setup, we can compare results for systems that have the same initial density and initial level of prevalence prior to culling.

We examine the dynamics exhibited by the model (eqq. [1][3]) when the population is subject to indiscriminate culling (i.e., an equal proportion is removed from each class in the model). We implement the culling regime as a discrete event that removes a fixed percentage of the population with continuous population regrowth between each cull event. Culling occurs at unit time intervals leading to $1 / d$ culls during the average lifetime of an individual in the absence of the disease. We run the culling regime for 30 consecutive periods of instantaneous cull followed by regrowth and examine the effect on the disease prevalence and the population density both during and at the end of this culling regime. In particular, we define the "resultant density" as the population density at the end of the 30 consecutive cull and subsequent regrowth events. Note that our results are qualitatively similar if we assume that culling occurs continuously rather than as a discrete event (see below and sec. A2 of the appendix). Our results are produced numerically using Matlab ordinary differential equation solvers, provided in a zip file, available online. ${ }^{1}$

In addition to assessing the impact of culling on the dynamics in the full model (eqq. [1]-[3]), we also develop a

1. Code that appears in The American Naturalist is provided as a convenience to readers. It has not necessarily been tested as part of peer review. 
model whose dynamics can respond to culling through demographic effects only:

$$
\frac{\mathrm{d} N}{\mathrm{~d} t}=b N(1-q N)-d N-\alpha p_{\mathrm{i}} N
$$

This "demographic-effects-only" model has the equivalent level of mortality to the full model (eqq. [1]-[3]) at the endemic steady state, but it cannot respond to changes in disease prevalence and therefore allows us to isolate the importance of changes to the disease dynamics as a result of culling. The parameters $b, d, q$, and $\alpha$ are the same as in the full model (eqq. [1]-[3]); $p_{\mathrm{i}}$ is the initial prevalence in the full model (which is constant in this model), and therefore prior to culling both models have the same steady-state density $N=N_{\mathrm{e}}$. Importantly, the density-dependent per capita birth rate, $b(1-q N)$, has an identical response in both models, and therefore any changes to the density in response to culling lead to the same change in the per capita birth rate.

We now note that the dynamics of the total density in the full model (found by summing eqq. [1]-[3]) can be written as

$$
\frac{\mathrm{d} N}{\mathrm{~d} t}=b N(1-q N)-d N-\alpha \frac{I}{N} N .
$$

Prior to culling the level of mortality is the same, since $p_{\mathrm{i}}=$ $I_{\mathrm{e}} / N_{\mathrm{e}}$. Critically, however, culling can lead to a change in the disease dynamics and therefore a change in disease prevalence $I / N$. This can lead to a change in the rate of mortality in the full model but not in the demographic effects model, since $p_{\mathrm{i}}$ does not change. A comparison of the full model and the demographic-effects-only model therefore allows us to determine the importance of changes due to the disease dynamics as a result of culling.

We also develop a "disease-effects-only" model that has the same disease dynamics as the full model (eqq. [1]-[3]) but has a fixed per capita birth rate $b\left(1-q N_{\mathrm{e}}\right)$, and therefore dynamic population density does not affect the rate of reproduction. This model is the same as equations (1)-(3) except for equation (1), which is modified as follows:

$$
\frac{\mathrm{d} S}{\mathrm{~d} t}=b N\left(1-q N_{\mathrm{e}}\right)-d S-\theta(I, N) S+\eta R .
$$

For DD transmission, the disease-effects-only model has the same endemic steady state, $N_{\mathrm{e}}$, as the full model, and therefore we can compare results between the disease-effects-only model and the full model to understand the contribution of demographic effects on compensatory growth. However, for FD transmission, the disease-effects-only model does not have a comparable nonzero endemic steady state, so this comparison is not valid. (In a similar manner, a model that cannot respond through disease or demographic effects cannot be compared with the full model, as culling would lead to population extinction.)
Analyzing the results for these models allows us to compare the compensatory growth following culling that is due to both demographic and disease effects (represented by the full model, eqq. [1]-[3]), demographic effects only (represented by eq. [6]), and disease effects only (represented by eq. [8]). The difference between the population densities in response to culling in these models allows us to partition compensatory growth due to disease dynamics only, namely, a population-level release from disease-induced mortality and compensatory growth due to demographic effects. In this study, we wish to understand the importance of compensatory growth due to a release from disease-induced mortality in response to culling for a range of key infection representations.

We examine the behavior of the model, equations (1)-(3), with DD and FD transmission for an SI framework (no recovery from infection) and for an SIR framework that represents lifelong immunity to infection. Later we consider an SIRS framework in which immunity can wane over time, targeted culling occurs on infected individuals only, and density-dependent mortality is included in addition to density-dependent reproduction. In addition to assessing the impact of culling in systems with classical modes of directly transmitted infection (DD and FD), we have also undertaken our analysis for systems with environmental, freeliving transmission (hereafter, FL transmission; Anderson and May 1981). The results for FL transmission are qualitatively similar to those with DD transmission, and general results are detailed in section A3 of the appendix. To emphasize the breadth of our findings, the results for systems with FL transmission are highlighted in the case study on the impact of culling on wild boar TB interactions.

\section{Results}

\section{The Effects of Culling in Populations with Virulent Infection and No Recovery}

Figure 1ai and 1aii shows that culling does not greatly decrease population density in systems with DD transmission in the absence of recovery. Indeed, after the initial culling events, the resultant density immediately prior to the next cull reaches a level exceeding the initial density $N_{\mathrm{e}}=1$. Moreover, the density can reach higher levels under a $25 \%$ cull than under a $10 \%$ cull. Here, culling leads to compensatory (even overcompensatory) growth as a result of changes to the disease dynamics. In particular, there is a reduction in the infected density and increase in susceptible density (fig. 1ai, 1aii), and therefore, as the total population density is not diminished, there is a reduction in disease prevalence (fig. 1ci, 1cii), which reduces the level of disease-induced mortality suffered by the population. The reduction in prevalence is greater under the higher level of culling, and so the compensatory 

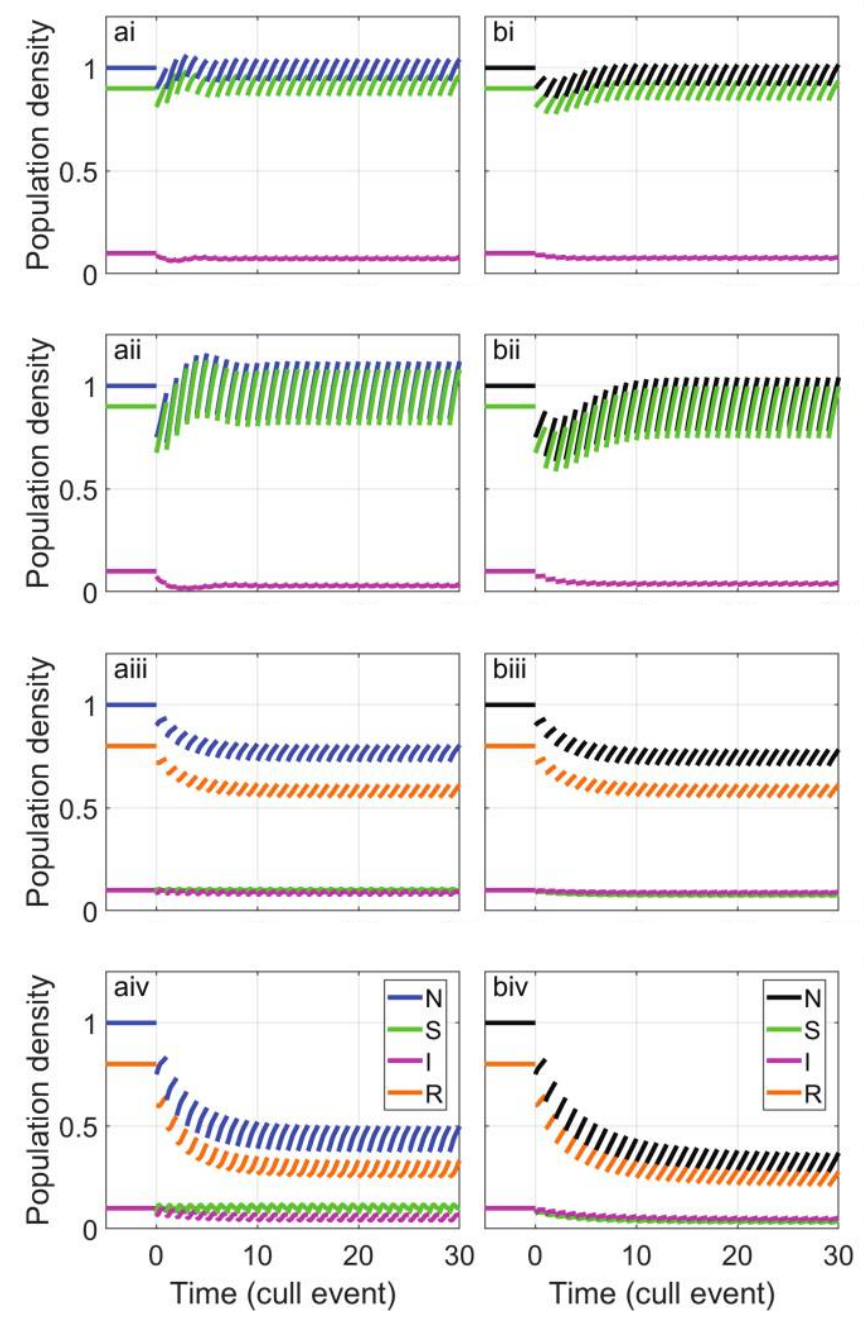
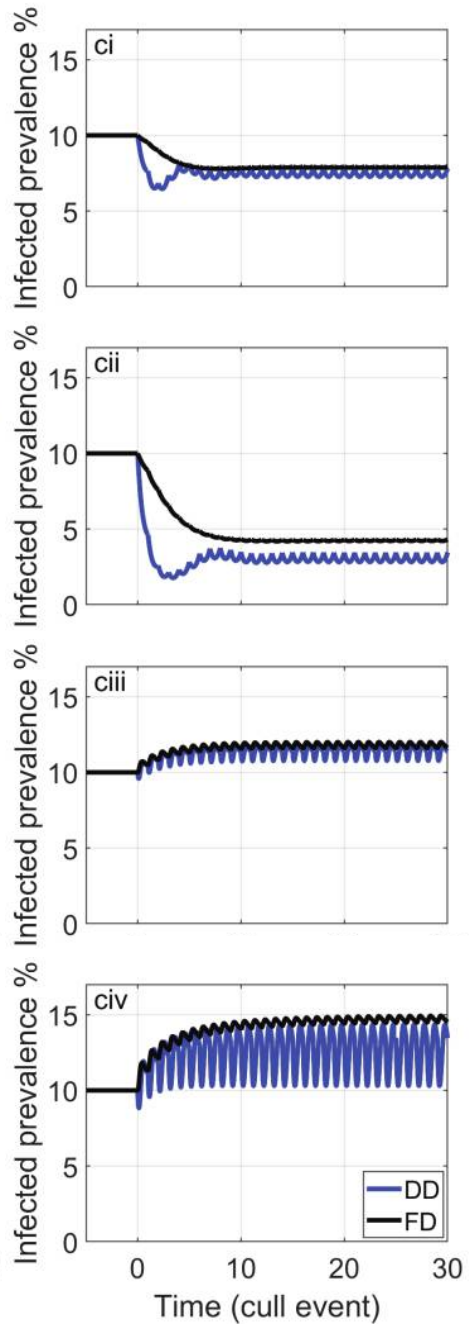

Figure 1: Population density and infected prevalence $(I / N)$ response to culling for equations (1)-(3). $i$ and $i i$ show results for the SI model, and $i i$ and $i v$ show results for the SIR model. Results are shown for a $10 \%$ cull $(i, i i i)$ and a $25 \%$ cull $(i i, i v)$. $a$ shows density-dependent (DD) disease transmission, and $b$ shows frequency-dependent (FD) disease transmission. $a$ and $b$ show the total density of susceptibles (green), the total density of infecteds (magenta), the total density of recovereds/immunes (orange), and the total population density (in $a$, blue for DD; in $b$, black for FD). $c$ shows the disease prevalence for DD (blue) and FD (black) transmission. Results are shown for a virulent infection, $\alpha=4$, with an initial endemic disease prevalence of $p_{\mathrm{i}}=10 \%$. Other parameters are $b=1.6, d=0.5$, and $\gamma=0$ for $i$ and $i$ and $\gamma=4$ for $i i i$ and $i v$.

growth due to infection processes is greater under higher culling. Under FD transmission the total population size increases less in response to culling (fig. $1 b i, 1 b i i$ ), but again there is a reduction in the infected density and increase in susceptible density that mitigates some of the mortality due to culling. The reduction in disease prevalence due to culling is smaller under FD than DD transmission (fig. 1ci, 1cii), which may explain the lower compensatory response.

To understand these findings more clearly, we examine the results for a $25 \%$ cull in population phase space (fig. 2ai, 2bi) and in terms of changes in the force of infection (fig. 2aii, 2bii). Under DD transmission the population response fol- lowing the initial culls is an increase in susceptibles but a decrease in infectives (fig. 2ai), since culling reduces the force of infection (fig. 2aii). Eventually, the density prior to the next cull stabilizes, with an increased density of susceptibles, a decreased level of infecteds, and a decreased force of infection. In this way, the increased mortality due to culling is compensated by population-level decreases in mortality due to the disease. Under FD transmission the population response following initial culls is an increase in susceptible and infected density (fig. 2bi). In particular, culling does not reduce the force of infection under FD transmission (fig. 2bii) as much, particularly in the initial culling events. The resultant popu- 

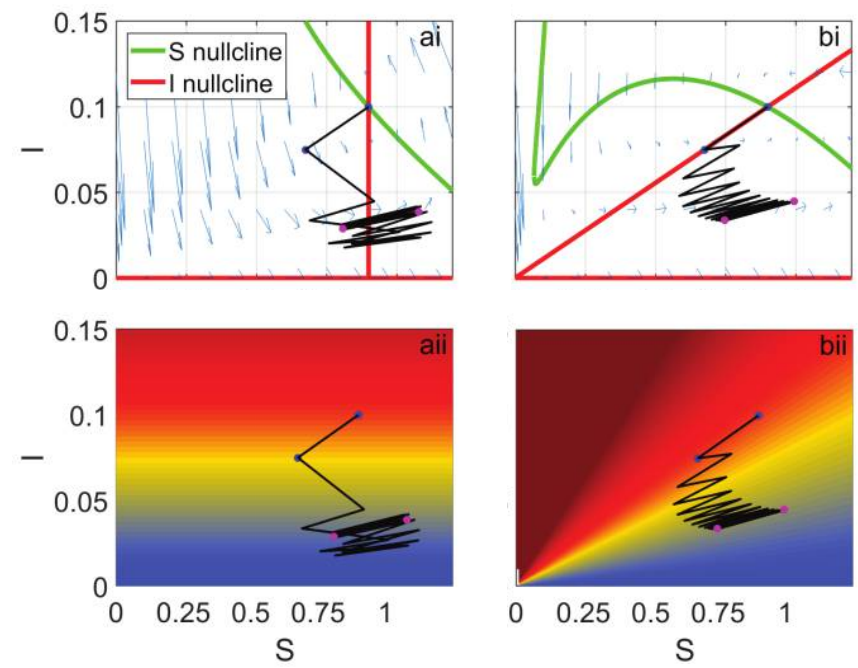

Figure 2: Population density response to repeated culling of $25 \%$ of the population for the SI model for density-dependent (DD; $a$ ) and frequency-dependent (FD; $b$ ) disease transmission. The figures show a population trajectory (solid black line) over the 30 cull events, with values immediately prior to and after the first cull highlighted with blue circles and those before and after the 30th cull highlighted with magenta circles. In $i$, the population trajectory is shown in phase space, with the red lines showing the boundary between regions where $I$ is decreasing and increasing and the green lines showing the boundary where $S$ is decreasing and increasing (as indicated by the flux arrows in the figures). In $i i$, the population trajectory is superimposed over the force of infection $\theta(I, N)$ with the color changing from dark blue to dark red as the force of infection increases. Results are shown for a virulent infection, $\alpha=4$, with an initial endemic disease prevalence of $p_{\mathrm{i}}=10 \%$. Parameters are as in figure 1 aii and 1 bii for DD and FD transmission, respectively.

lation therefore supports a higher level of infecteds compared with DD transmission, and therefore while the compensatory effects due to reduced population-level disease-induced mortality are still significant, they are smaller under FD transmission.

The compensatory population growth in response to culling can result from two mechanisms: (1) a reduction in the impact of density dependence on reproduction and (2) a population-level reduction in disease-induced mortality due to changes in the disease dynamics. Both of these mechanisms could occur for the population-level response to culling for our full model, equations (1)-(3). In figure 3ai, 3aii, $3 b i$, and $3 b i i$ we compare the results from the full model with the demographic-effects-only model (eq. [6]), and in fig. $3 b i$ we compare the results from the full model with the diseaseeffects-only model (eq. [8]), noting that this latter comparison is valid only for DD transmission. The difference between the full model and the disease-effects-only model represents the compensation that is solely due to demographic effects, and here this compensation is minimal (fig. 3bi). The difference between the full model and the demographic-effects-only model represents the compensation due to the populationlevel reduction in disease-induced mortality and accounts for most of the compensatory growth (fig. 3bi, 3bii). A key result is that compensation due to disease effects in response to culling can be substantial under both DD and FD transmission.

\section{The Effects of Culling in Populations with Virulent Infection and Recovery to Immunity}

Figure 1aiii, 1aiv, 1biii, and 1biv shows that compensatory growth due to a population-level release from disease-induced mortality is not evident in systems with lifelong immunity following infection. Here, culling leads to a significant reduction in the population density and an increase in infected prevalence (fig. 1ciii, 1civ). This effect is most pronounced under FD transmission, as here the force of infection remains high when the population abundance is reduced. In systems with lifelong immunity, the population composition in the absence of culling includes a relatively large proportion of recovered/immune individuals. Culling removes all classes equally, but it takes time for individuals to move through the infection stages to reach the recovered class; therefore, culling leads to a larger relative reduction to the recovered class density, and so the proportion of the population that suffers virulence $(I / N)$ increases. Figure $4 a i$ and $4 a i i$ shows that culling leads to a greater reduction in population density in the full SIR model than in the demographic-effects-only model. Also, for DD transmission (fig. 4bi) culling decreases population density less in the full SIR model than in the disease-effects-only model. Therefore, culled populations that support virulent infections with recovery to lifelong immunity do not benefit from reduced disease-induced mortality but, as shown here for DD transmission, do exhibit 

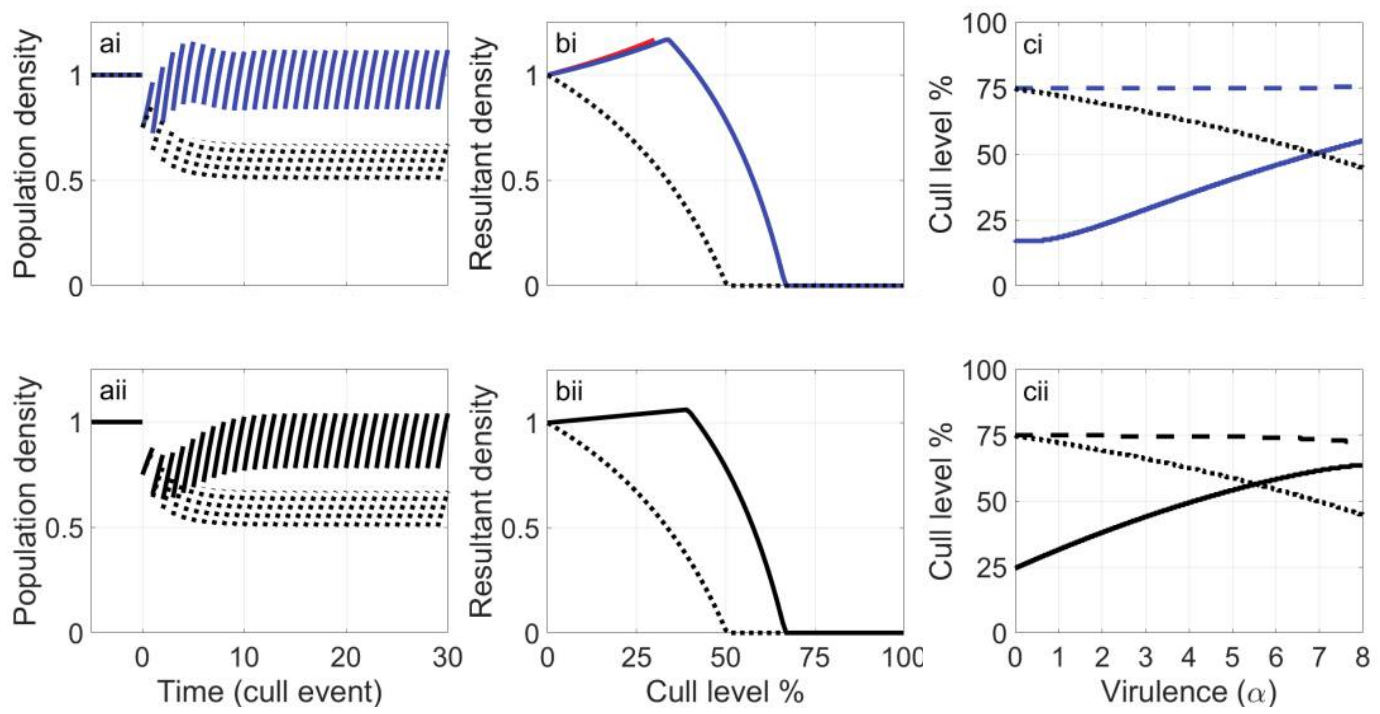

Figure 3: Population density compensatory response to culling for the SI model. $a$, Population density response to repeated culling of $25 \%$ of the population for the full model, equations (1)-(3), under SI $(\gamma=0)$ dynamics for density-dependent (DD; $i)$ and frequency-dependent (FD; ii) disease transmission (DD, blue; FD, black) and for the demographic-effects-only model (dotted). $b$, Resultant population density at the end of sequential cull and subsequent regrowth periods for different levels of culling for the full model with DD ( $i$; blue) and FD ( $i i$; black) transmission, the demographic-effects-only model (dotted), and, in bi, the disease-effects-only model (red). Note that this red line is valid only for culling levels less than 30\%, with higher culling levels leading to disease and population extinction. The difference between the solid blue and the dotted line in $b i$ and the difference between the solid black and the dotted line in bii represent the amount of compensation due to the disease effects, and in $b i$ the difference between the red line and the blue line represents the compensation due to demographic effects. $c$, Plot of virulence against the level of culling required to eradicate the infection (solid line) and the population (dashed line) as well as the level of culling required to eradicate the population in the demographic-effects-only model (dotted line) for DD (i) and FD (ii) transmission. Results are shown for a virulent infection, $\alpha=4$ (except in $c$, where $\alpha$ varies), with an initial endemic disease prevalence of $p_{\mathrm{i}}=10 \%$. Other parameters are as in figure 1.

compensation due to demographic effects, which mitigate some of the mortality due to culling. A key point is that when there is lifelong immunity there can be a significant reduction in the population density, and the disease can make the population less resilient to harvesting.

\section{The Impact of Culling on Population Management}

Culling is often used as a strategy for population eradication, for instance, to remove pest or invasive species. We can use our model to investigate how the presence of virulent infection changes the level of culling required to eradicate a population. Figure $3 b i$ and $3 b i i$ shows that in systems without lifelong immunity the compensatory effects due to changes in the disease dynamics mean that an increased level of culling is required to eradicate the population. In systems that have lifelong immunity (fig. $4 b i, 4 b i i$ ), the presence of virulent disease makes the population harder to eradicate under DD transmission but easier to eradicate under FD transmission. Here, under FD transmission, culling leads to an increase in infection prevalence and so increases the population disease- induced mortality in addition to culling mortality. In contrast, under DD transmission, high levels of culling reduce the proportion of recovered individuals to such an extent that the system acts like one in which lifelong immunity is absent. Our work therefore indicates that programs to eradicate invasive species will be hindered if the invasive species harbors a virulent nonimmunizing parasite or immunizing parasite under DD transmission but facilitated for strongly immunizing virulent parasites with FD transmission. It is therefore critical to understand the nature of transmission and immunity to the key virulent infectious diseases of a target species prior to the use of culling for population elimination.

\section{The Impact of Culling on Disease Management}

Culling is also used as a strategy to manage or eradicate a disease. Here, the goal may be to eliminate the disease while maintaining viable or maximum levels of host density (Davidson et al. 2009; Beeton and McCallum 2011; Hallam and McCracken 2011; Boadella et al. 2012), or the impact on the 

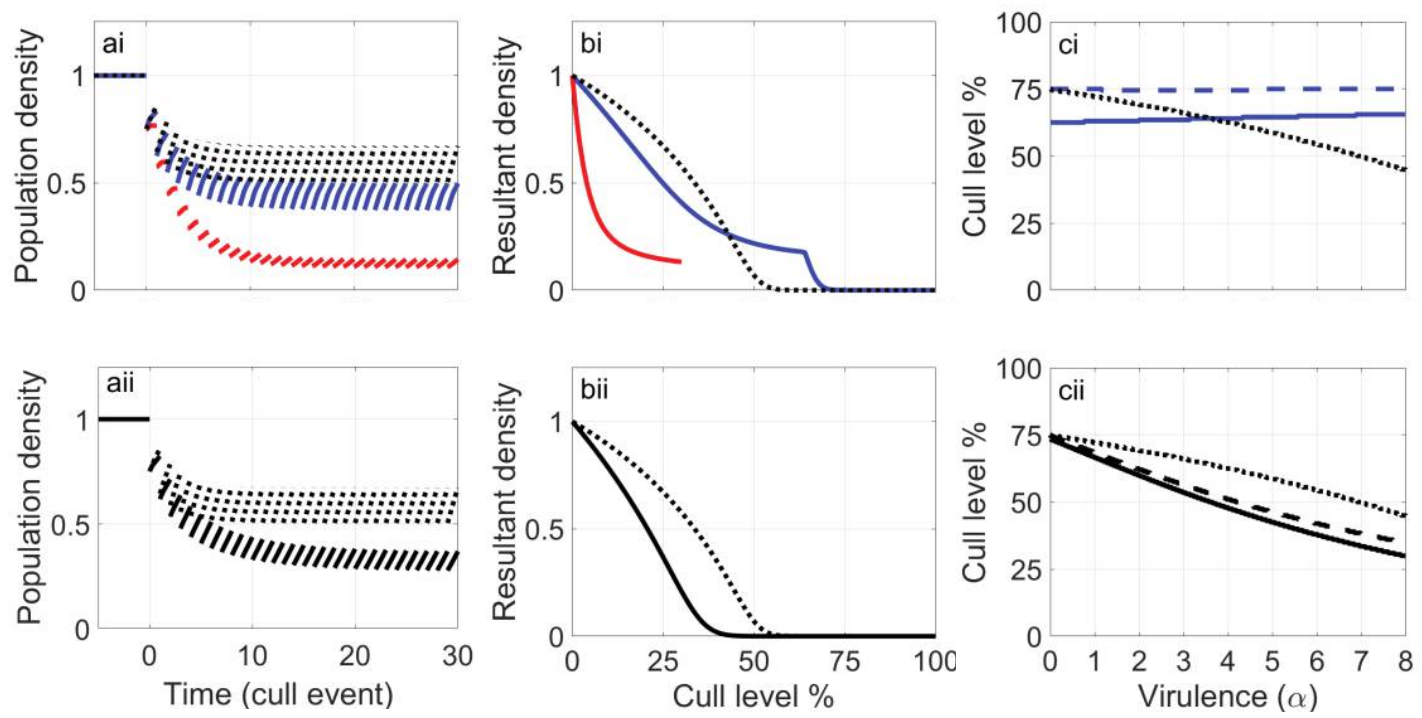

Figure 4: Population density compensatory response to culling for the SIR model. $a$, Population density response to repeated culling of $25 \%$ of the population for the full model, equations (1)-(3), under SIR $(\gamma=4)$ dynamics for density-dependent (DD; $i)$ and frequency-dependent (FD; ii) disease transmission (DD, blue; FD, black), for the demographic-effects-only model (dotted) and for the disease-effects-only model (red). Here, the presence of the disease leads to a lower population in response to culling. $b$, Resultant population density at the end of sequential cull and subsequent regrowth periods for different levels of culling the full model with DD (i) and FD (ii) transmission (DD, blue; FD, black), the demographic-effects-only model (dotted), and the disease-effects-only model (red). Note that this red line is valid only for culling levels less than $30 \%$, with higher culling levels leading to disease and population extinction. The difference between the solid blue and the dotted line in $b i$ and the difference between the solid black and the dotted line in bii represent the amount of compensation due to the disease effects, and in $b i$ the difference between the red line and the blue line represents the compensation due to demographic effects. $c$, Plot of virulence against the level of culling required to eradicate the infection (solid line) and the population (dashed line) as well as the level of culling required to eradicate the population in the demographic-effects-only model (dotted line) for DD (i) and FD (ii) transmission. Results are shown for a virulent infection, $\alpha=4$ (except in $c$, where $\alpha$ varies), with an initial endemic disease prevalence of $p_{\mathrm{i}}=10 \%$. Other parameters are as in figure 1.

host density is of less concern (O’Brien et al. 2011). Our study indicates that the level of culling required to eradicate the disease increases as the virulence increases for DD transmission under both the SI and SIR model frameworks (figs. 3ci, $4 c i$ ). Under FD transmission the level of culling required to eradicate the disease increases in the absence of immunity and decreases in the presence of lifelong immunity (figs. 3cii, 4cii). A key result is that the interval between the level of culling required for disease eradication and population extinction is narrow at high virulence in the absence of immunity and narrow at all levels of virulence with immunity. These results highlight the importance of understanding the infection status of a population before culling for disease management, as the level of culling required for disease eradication may put the population at risk of extinction.

\section{Generality of Model Findings}

Our analysis has shown how culling can lead to positive compensatory growth due to a population reduction in diseaseinduced mortality in systems without immunity and can lead to larger decreases in population density in systems with life- long immunity. It is therefore important to ascertain the threshold in the level of immunity that partitions the positive and negative impacts on growth in response to culling. To do this, we examine how the long-term density responds to culling in an SIRS model in which infection leads to immunity but where immunity can wane. For both DD and FD transmission, positive compensatory growth due to infection feedbacks in response to culling occurs except when the recovery rate is high and the loss of immunity is low (fig. 5). Therefore, our results indicate that culling can lead to a population-level reduction in disease-induced mortality that mitigates the impact of culling on population abundance, provided the infection does not lead to long-lasting immunity.

We confirm our findings for discrete culling in a model of continuous culling (the detailed analysis is presented in secs. A2 and A4 of the appendix). The results for the continuous-cull model approximate the average density of those for the equivalent discrete cull (figs. A1, A3, A4). As such, the model for continuous culling exhibits the compensation due to a release from disease-induced mortality that we are investigating, although as an average it does not show the potential increase in resultant density above the endemic 

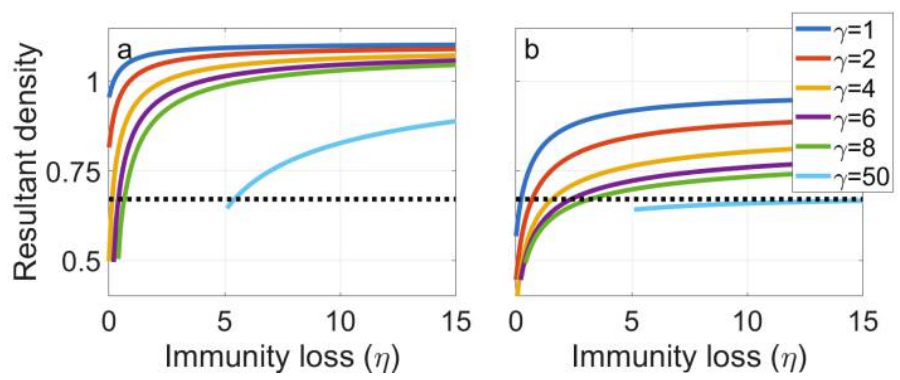

Figure 5: Resultant population density after 30 sequential cull and subsequent regrowth periods culling $25 \%$ of the population for the SIRS model (eqq. [1]-[3]) plotted against waning immunity, $\eta$, for different levels of recovery, $\gamma$, for density-dependent $(a)$ and frequency-dependent (b) disease transmission. The dotted line represents the resultant population density for the demographic-effects-only model. The difference between the solid lines and dotted line represents the positive or negative compensatory effect due to changes in the disease dynamics. Results are shown for an initial endemic disease prevalence of $p_{\mathrm{i}}=10 \%$. Other parameters are as in figure 1 . Truncated results indicate parameter levels that do not satisfy requirements for valid solutions (eqq. [A8], [A9]).

steady state illustrated in figure 3. However, it does allow a robust comparison of steady states for our different model formulations. In particular, we compare the steady states for total density in the full model for DD and FD transmission - $N_{\mathrm{DD}}$ and $N_{\mathrm{FD}}$, respectively — with the demographiceffects-only model, $N_{\text {dem }}$, and the disease-effects-only model, $N_{\text {dis }}$ and we compare these values with the density prior to culling, $N_{\mathrm{e}}$. For the SI model, we show that for DD transmission $N_{\mathrm{e}}>N_{\text {DD }}>N_{\text {dis }}>N_{\text {dem }}$ and that for FD transmission $N_{\mathrm{e}}>N_{\mathrm{FD}}>N_{\text {dem }}$, which confirms the findings of figure 3 and figure A2i. In our illustrated results (fig. A2ai), we see that $N_{\text {DD }}-N_{\text {dem }} \gg N_{\text {DD }}-N_{\text {dis }}$, and therefore the release from disease-induced mortality contributes most of the compensation in response to culling (a similar result holds for FD transmission; fig. A2bi). These results also hold for the SIRS model when there is a low rate of recovery or a high rate of loss of immunity. When immunity is sufficiently long-lived (e.g., high recovery and low loss of immunity), for DD transmission $N_{\mathrm{e}}>N_{\text {dem }}>N_{\text {DD }}>N_{\text {dis }}$ and for FD transmission $N_{\mathrm{e}}>N_{\mathrm{dem}}>N_{\mathrm{FD}}$, which again confirms the findings in figure 4 and figure A2ii. These analytical results hold for all valid parameter values and confirm our key finding that the compensation due to disease effects exceeds those due to demographic effects in systems without long-lived immunity.

We also examine the impact of culling that is targeted on infected individuals and find that compensation due to changes in the disease dynamics still occurs under the SI model but is greater under FD than DD transmission (see sec. A4 of the appendix and fig. A7). The amplified compensatory growth under FD transmission occurs because targeted culling leads to a direct decrease in the force of infection, since infected density decreases more rapidly than total density (under indiscriminate culling, infected and total density decrease at the same rate due to culling). For the SIR model, targeted culling has little effect on density and prevalence and therefore the negative impacts of culling and disease are no longer observed, but the level of compensation is minimal. For the SIR model, targeted culling does initially reduce the force of infection, but as a consequence fewer individuals progress to the recovered and immune class; overall, these two effects balance. In general, the findings for the model with targeted culling confirm our previous results that compensation due to disease effects occurs in the absence of long-lasting immunity. We also compare the steady states for total density under continuous targeted infected culling for DD and FD transmission, $N_{\mathrm{DD}}^{\mathrm{T}}$ and $N_{\mathrm{FD}}^{\mathrm{T}}$, respectively. We show that for a sufficient level of targeted infected culling and low rate of recovery or high rate of loss of immunity, $N_{\mathrm{FD}}^{\mathrm{T}}>N_{\mathrm{DD}}^{\mathrm{T}}>N_{\mathrm{e}}$, confirming the findings in figures $\mathrm{A} 7$ and A8 and supporting our finding of the potential for targeted infected culling to induce an overcompensatory population growth response.

The full model (eqq. [1]-[3]) includes demographic crowding effects on birth only. In section A5 of the appendix, we examine the impact of culling on compensatory growth for a version of the full model that can include density-dependent birth and/or death. The results are unchanged under DD transmission regardless of whether density dependence is on birth, death, or a combination of both. Under FD transmission the compensatory effect due to a release from disease mortality decreases as the level of density-dependent death increases relative to density-dependent birth. The only scenario in which there is no compensatory effect due to changes in disease dynamics is when there is purely density-dependent death (under FD transmission). Therefore, our key finding that culling can lead to compensatory growth due to changes in the disease dynamics is evident for almost all scenarios of density-dependent birth and death. An analytical explanation of these findings is presented in section A5 of the appendix. A parameter sensitivity analysis examining how the 
level of compensatory growth varies with disease virulence, initial prevalence, and cull period is presented in section A6 of the appendix.

\section{Case Study}

We highlight the applicability of our findings by considering a case study of the use of culling to manage TB in Eurasian wild boar (Sus scrofa) in central Spain. Here, environmental drivers, such as summer drought, can lead to aggregation with associated high prevalence of infection of Mycobacterium tuberculosis complex (MTC), which are the causative agents of animal TB (Vicente et al. 2007). We assume that the driver of infection in the wild boar TB system is through environmental contact with a free-living MTC pathogen, which is shed from the most infectious individuals (Barasona et al. 2017). It is appropriate to assume that the population is well mixed in terms of transmission, as on managed estates infection is likely to occur at scarce water holes where freeliving MTC can persist and which are utilized frequently by the whole population. Therefore, the FL transmission mode is used in this case study (see sec. A3 of the appendix and recall that FL transmission produces qualitatively similar findings to DD transmission). In central Spain wild boar are the primary reservoir host for MTC, and in some regions up to $70 \%$ of the population can be infected with MTC, of which half (35\% of the total population) may exhibit generalized infection (infected and infectious; Vicente et al. 2013; Santos et al. 2015). Individuals with generalized infection suffer high levels of disease-induced mortality (Barasona et al. 2017). Since wild boar have an economic and cultural value for the hunting community in Spain, there has been a reluctance to use additional culling to control $\mathrm{TB}$, as it may result in decreased population abundance. However, since the wild boar TB system is characterized by high disease-induced mortality and no recovery from infection (Barasona et al. 2016), our earlier general results indicate that culling could result in compensatory growth due to reduced disease-induced mortality offsetting the mortality associated with culling and thus sustaining population abundance.

We extend our model framework to represent the wild boar TB systems for a single geographical managed estate containing a homogeneously mixed population covering an area $3 \times 3 \mathrm{~km}^{2}$ in size. The population density of wild boar is separated into different age classes to capture distinct disease and reproductive characteristics for piglets (aged $0-1$ year), yearlings (aged 1-2 years), and adults (aged $\geq 2$ years). Furthermore, the age classes are split into susceptible, infected, and generalized (infected and infectious) classes to reflect disease status. Yearlings and adults can give birth, and in contrast to our model formulation in equations (1)-(3), the crowding parameter $q$ (see section A7.1 of the appendix), used to limit the disease-free total population density to the carrying capacity, is independent of the endemic disease prevalence. Infection occurs through environmental contact with a freeliving MTC pathogen that is shed from individuals with the generalized infection. The population dynamics of wild boar and $\mathrm{TB}$ are represented by a system of differential equations that are an extension of our general framework, equations (1)(3). Full details of the model and parameterization can be found in section A7 of the appendix.

When there is an indiscriminate cull on yearlings and adults, the population density shows an initial drop followed by an increase, with peak density falling by only $10 \%$ in response to a $25 \%$ annual cull (fig. $6 a i$ ). While total population size shows only a small reduction, there is a more significant reduction in infected individuals, and total prevalence drops from $64 \%$ to $43 \%$ and generalized prevalence drops from $35 \%$ to $22 \%$. More generally (fig. $6 b i, 6 c i$ ), there is only a shallow decline in population density in response to increased culling up to a threshold at which the disease is eradicated from the system (50\% cull). After this, the population level declines rapidly. When there is a targeted annual cull of $25 \%$ of generalized yearlings and adults (fig. 6ii), we see an increase in the total population but only a modest decrease in prevalence and in particular little change in the density of infected and generalized individuals. These results highlight the fact that compensatory growth due to reduced disease-induced mortality may offset increased culling and may lead to a reduction in TB prevalence in wild boar without detrimental reductions in density. Our general predictions may therefore be applicable in this system and highlight the importance of detailed modeling in the context of culling in the face of disease.

\section{Discussion}

Despite the ubiquity of infectious disease in nature, there has been little work on the impact of disease on harvested populations. Our key result is that population reductions from culling and harvesting are compensated in a wide range of infectious disease scenarios due to a population-level release from disease-induced mortality. The compensatory effect increases as disease virulence, the preculling level of prevalence, and the level of culling increase and occurs for systems with $\mathrm{DD}, \mathrm{FD}$, and environmental (FL) modes of transmission. The key outcome is that culling in systems that harbor virulent parasites can lower disease prevalence without significantly reducing - or indeed increasing - population density. The population can therefore be regulated at a similar density by disease or at reduced prevalence by a combination of culling and disease.

Compensation due to changes in disease dynamics occurs in the absence of long-lasting immunity. With long-lasting immunity and indiscriminate culling, disease generally increases the impact of culling and harvesting, reducing the 

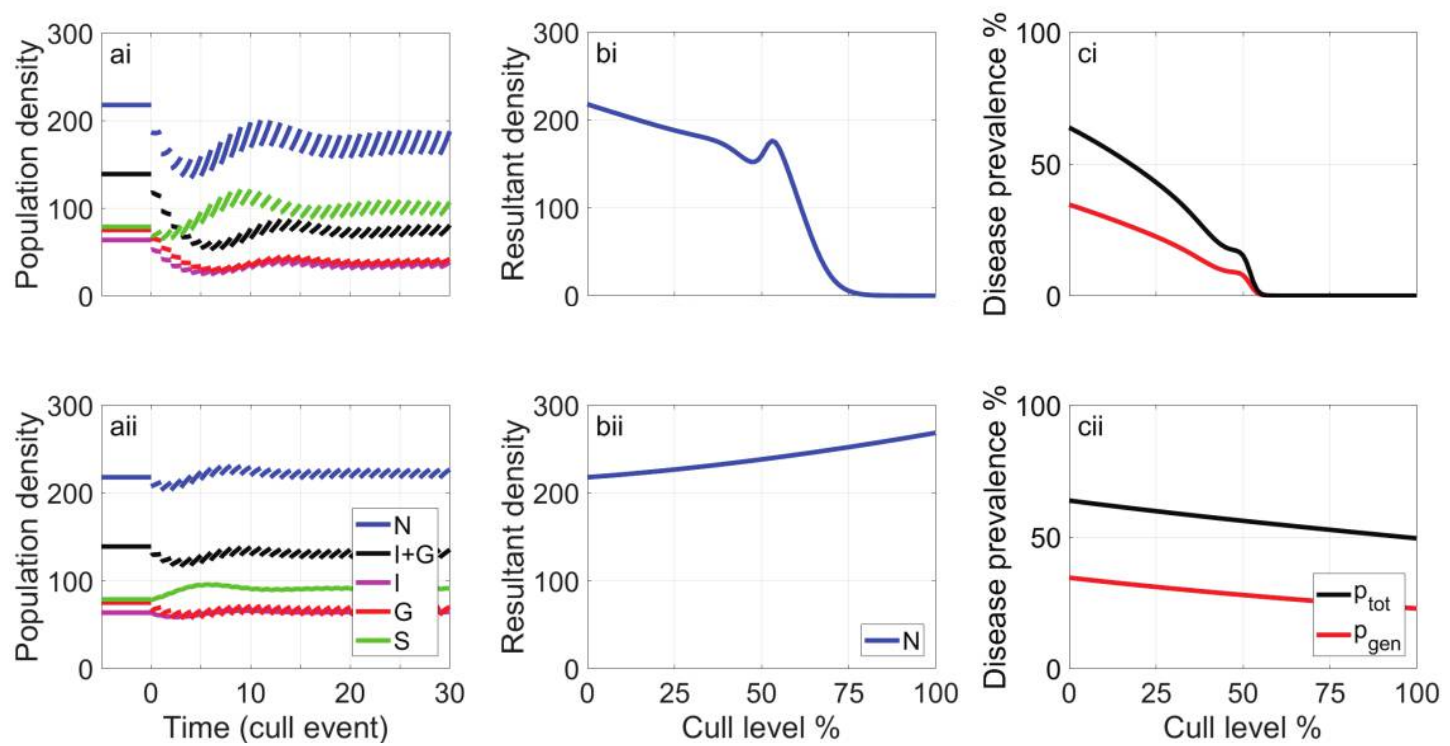

Figure 6: Results for the wild boar tuberculosis (TB) model in response to 30 cull events of $25 \%$ for indiscriminate culling of the yearling and adult population $(i)$ and targeted culling of generalized yearlings and adults (ii). The population dynamics over time are shown in $a$ for total density (blue), infected and generalized density (black), susceptible density (green), infected density (magenta), and generalized density (red). The initial population assumes a TB prevalence, $(I+G) / N$, of $64 \%$ and a generalized prevalence, $G / N$, of $35 \%$. $b$ shows the resultant total population (blue) and $c$ shows the total prevalence (black) and generalized prevalence (red) after 30 sequential cull and subsequent regrowth periods for different levels of hunting. The model and its parameters are outlined in figure A7.

population density compared with systems without the disease. Although there are examples of lifelong immunity in wildlife and livestock populations (rinderpest vaccine produces lifelong immunity in African cattle; Roeder et al. 2013), there are also many examples, including $\mathrm{TB}$, where vaccinederived immunity wanes (Thom et al. 2012). This indicates that even in those diseases with acquired immunity, this protection may often be partial or wane, leading to an SIRS model where individuals become susceptible again after a period of immunity. It is therefore likely that many wildlife systems that support virulent infectious disease will exhibit compensatory growth due to reduced disease-induced mortality following culling. Of course, many populations will have multiple diseases, but the key point is to understand the overall disease burden and in particular whether there is widespread immunity to the key sources of virulence. System-specific models can then determine whether the infectious disease allows increased exploitation or makes the host population more vulnerable. For example, our system-specific model of the wild boar TB interaction in central Spain predicted a strong effect of compensation due to changes in disease dynamics leading to only modest reductions in the population abundance due to hunting. This is, therefore, an example in which the impact of harvesting is offset in a host that harbors a virulent parasite and suggests that hunting is likely to be a sustainable management option in this system.
Our results have important consequences for the use of culling to manage infectious disease. The impact of harvesting on wildlife disease has been previously considered in models with long-lasting immunity (Choisy and Rohani 2006; Bolzoni et al. 2007), which reported an increase in prevalence. Our results confirm these findings, since in systems with long-lasting immunity harvesting will reduce the density of immune individuals to a greater proportional extent than other classes (Bolzoni et al. 2007; McCallum 2016). We also support previous studies (Barlow 1996; Wasserberg et al. 2009; Potapov et al. 2012; Storm et al. 2013) that showed that indiscriminate culling is more effective at reducing disease prevalence when infection results from DD rather than FD transmission. However, we show that targeted culling is more effective when transmission is FD. System-specific models have shown how localized culling could reduce the prevalence of classical swine fever in wild pigs (Cowled et al. 2012) and reduce the prevalence and spread of chronic wasting disease in white-tailed deer (Storm et al. 2013; Potapov et al. 2016), predictions that are supported by observations in the field (Carstensen et al. 2011; Manjerovic et al. 2014).

Our results highlight the difficulty of using culling to eradicate an infectious disease and may explain empirical findings that suggest that culling is not an effective disease management tool. For example, bovine TB has persisted in badger populations in Great Britain despite comprehensive culling 
campaigns (Donnelly et al. 2006); Gortázar et al. (2015) reviewed culling programs worldwide and reported few that achieved $100 \%$ efficacy. Theoretical models have suggested that culling could not control white-nose syndrome in bats (Hallam and McCracken 2011), that very high levels of culling were required to eradicate paratuberculosis in rabbits (Davidson et al. 2009) and Tasmanian devil facial tumor disease (Beeton and McCallum 2011), and that culling may increase disease transmission through changes in other ecologically driven factors (Prentice et al. 2014). While our findings indicate that culling can maintain prevalence at reduced levels, they also highlight that high levels of culling are required to eradicate an infection and that there is a narrow range of culling levels between disease eradication and population extinction. System-specific models are therefore required to determine the likelihood of success and the risk of population extinction that may result from culling programs to control disease.

Previous model studies of the wild boar TB system suggested that culling may contribute to TB management when used in conjunction with other control measures (Anderson et al. 2013). Our model of TB and wild boar shows how such system-specific models can be built to understand when and how culling can be used as a management tool in wildlife systems that harbor virulent disease. Wild boar hunting is a source of income, while in some localities spillover of TB into livestock has economic impacts. Our results show that hunting could be a viable method for controlling TB in wild boar because hunting leads to a significant drop in disease prevalence with the model results supported by observations in central Spain (Boadella et al. 2012; Barasona et al. 2016). This is a win-win situation for managed estates since in addition to a decrease in disease prevalence a large proportion of the mortality from hunting is countered by a reduction in disease-induced mortality. The model results indicate that the largest decrease in prevalence and density of infectious individuals is for indiscriminate culling (of juveniles and adults). Here, there is a threshold at which culling eradicated the disease ( $60 \%$ in our model study), after which population abundance decreases rapidly, leading to extinction when culling reaches $75 \%$. It may therefore be possible to eradicate TB in wild boar through culling, but it would be critical to determine these thresholds at a regional level. Targeted culling of infectious wild boar resulted in only modest reductions in prevalence and no discernible change in the density of infecteds. This may explain the failure of targeted culling to control TB in empirical studies (Che'Amat et al. 2016).

Overcompensatory population regrowth in response to culling events is well known in systems that do not consider infectious disease; Abrams and Matsuda (2005) termed this a "hydra effect." Abrams (2009) outlined three possible mechanisms that may produce the hydra effect: (1) additional mortality altering preexisting population oscillations in a way that leads to an increased density, (2) a temporal separation of mortality and density dependence, and (3) mortality of a consumer leading to overcompensatory changes in other aspects in the food web. Our results for the model with discrete culling show that the resultant density can exceed the original density. Our results with targeted infected culling, for both discrete and continuous model setups, show that the resultant density may also surpass the total population density in the absence of culling. These results arise as culling induces population regrowth in an environment with reduced prevalence and therefore reduced disease-induced mortality. Therefore, our novel insight is that the release from mortality caused by endemic disease following culling can also lead to a hydra effect. This has similarities to the hydra effect due to the consumer-resource mechanism (Abrams 2009, mechanism iii), where additional mortality of the consumer leads to a reduction in mortality for the resource. Our scenario is different in that it occurs within a single species.

Our key finding is that mortality due to culling in systems that harbor virulent infections may be compensated by reductions in disease-induced mortality. We have also demonstrated that it is important to fully understand the infection processes and to model the specific system before using culling as a disease management tool (Beeton and McCallum 2011). Given the ubiquity of disease and the importance of harvested populations for food security, the novel compensatory mechanism we have identified may help to provide sustainable management of these populations.

\section{Acknowledgments}

This is a contribution to Ministerio de Economía y Competitividad (MINECO) Plan Nacional (grant WILD DRIVER ref. CGL2017-89866 and the European Regional Development Fund [EU-FEDER]). E.T. was supported by the Maxwell Institute Graduate School in Analysis and its Applications, a Centre for Doctoral Training funded by the United Kingdom Engineering and Physical Sciences Research Council (grant EP/L016508/01), the Scottish Funding Council, Heriot-Watt University, and the University of Edinburgh. We thank Y. Michalakis, B. Bolker, A. Hurford, and an anonymous referee for helpful comments and guidance in preparing the manuscript.

\section{Literature Cited}

Abrams, P. A. 2009. When does greater mortality increase population size? The long history and diverse mechanisms underlying the hydra effect. Ecology Letters 12:462-474.

Abrams, P. A., and H. Matsuda. 2005. The effect of adaptive change in the prey on the dynamics of an exploited predator population. Canadian Journal of Fisheries and Aquatic Sciences 62:758-766. 
Anderson, L., C. Gortázar, J. Vicente, M. Hutchings, and P. White. 2013. Modelling the effectiveness of vaccination in controlling bovine tuberculosis in wild boar. Wildlife Research 40:367-376.

Anderson, R., and R. May. 1979. Population biology of infectious diseases 1. Nature 280:361-367.

1981. The population dynamics of microparasites and their invertebrate hosts. Philosophical Transactions of the Roval Societv B 291:451-524.

Barasona, J., P. Acevedo, I. Díez-Delgado, J. Queiros, R. CarrascoGarcía, C. Gortázar, and J. Vicente. 2016. Tuberculosis-associated death among adult wild boars, Spain, 2009-2014. Emerging Infectious Diseases 22:2178-2180.

Barasona, J., J. Vicente, I. Díez-Delgado, J. Aznar, C. Gortázar, and M. Torres. 2017. Environmental presence of Mycobacterium tuberculosis complex in aggregation points at the wildlife/livestock interface. Transboundary and Emerging Diseases 64:1148-1158.

Barlow, N. 1996. The ecology of wildlife disease control: simple models revisited. Journal of Applied Ecology 33:303-314.

Beeton, N., and H. McCallum. 2011. Models predict that culling is not a feasible strategy to prevent extinction of Tasmanian devils from facial tumour disease. Journal of Applied Ecology 48:1315-1323.

Boadella, M., J. Vicente, F. Ruiz-Fons, J. de la Fuente, and C. Gortázar. 2012. Effects of culling Eurasian wild boar on the prevalence of $M y$ cobacterium bovis and Aujeszky's disease virus. Preventive Veterinary Medicine 107:214-221.

Bolzoni, L., L. Real, and G. De Leo. 2007. Transmission heterogeneity and control strategies for infectious disease emergence. PLoS ONE 2:e747.

Carstensen, M., D. J. O’Brien, and S. Schmitt. 2011. Public acceptance as a determinant of management strategies for bovine tuberculosis in free-ranging US wildlife. Veterinary Microbiology 151:200-204.

Che'Amat, A., J. A. Armenteros, D. Gonzalez-Barrio, J. F. Lima I. Díez-Delgado, J. A. Barasona, B. Romero, K. P. Lyashchenko, J. A. Ortiz, and C. Gortázar. 2016. Is targeted removal a suitable means for tuberculosis control in wild boar? Preventive Veterinary Medicine 135:132-135.

Choisy, M., and P. Rohani. 2006. Harvesting can increase severity of wildlife disease epidemics. Proceedings of the Roval Societv B 273 2025-2034.

Cowled, B., M. Garner, K. Negus, and M. Ward. 2012. Controlling disease outbreaks in wildlife using limited culling: modelling classical swine fever incursions in wild pigs in Australia. Veterinarv Research 43:3.

Davidson, R., G. Marion, P. White, and M. Hutchings. 2009. Use of host population reduction to control wildlife infection: rabbits and paratuberculosis. Epidemiology and Infection 137:131-138.

Díez-Delgado, I., I. A. Sevilla, B. Romero, E. Tanner, J. A. Barasona, A. R. White, P. W. Lurz, et al. 2018. Impact of piglet oral vaccination against tuberculosis in endemic free-ranging wild boar populations. Preventive Veterinary Medicine 155:11-20.

Donnelly, C. A., R. Woodroffe, D. Cox, F. J. Bourne, C. Cheeseman, R. S. Clifton-Hadley, G. Wei, et al. 2006. Positive and negative effects of widespread badger culling on tuberculosis in cattle. Nature 439: 843-846.

Gortázar, C., I. Díez-Delgado, J. A. Barasona, J. Vicente, J. de la Fuente, and M. Boadella. 2015. The wild side of disease control at the wildlifelivestock-human interface: a review. Frontiers in Veterinary Science 1:27.

Hallam, T., and G. McCracken. 2011. Management of the panzootic white-nose syndrome through culling of bats. Conservation Biology 25:189-194.
Keeling, M. J., and P. Rohani. 2008. Modeling infectious diseases in humans and animals. Princeton University Press, Princeton, NJ.

Manjerovic, M., M. Green, N. Mateus-Pinilla, and J. Novakofski. 2014. The importance of localized culling in stabilizing chronic wasting disease prevalence in white-tailed deer populations. Preventive Veterinary Medicine 113:139-145.

McCallum, H. 2016. Models for managing wildlife disease. Parasitology 143:805-820.

O’Brien, D. J., S. M. Schmitt, B. A. Rudolph, and G. Nugent. 2011. Recent advances in the management of bovine tuberculosis in freeranging wildlife. Veterinary Microbiology 151:23-33.

Potapov, A., E. Merrill, and M. Lewis. 2012. Wildlife disease elimination and density dependence. Proceedings of the Roval Societv B 279: 3139-3145.

Potapov, A., E. Merrill, M. Pybus, and M. Lewis. 2016. Chronic wasting disease: Transmission mechanisms and the possibility of harvest management. PLoS ONE 11:e0151039.

Prentice, J. C., G. Marion, P. C. White, R. S. Davidson, and M. R. Hutchings. 2014. Demographic processes drive increases in wildlife disease following population reduction. PloS ONE 9:e86563.

Ricker, W. 1954. Stock and recruitment. Lournal of the Fisheries Board of Canada 11:559-623.

Roeder, P., J. Mariner, and R. Kock. 2013. Rinderpest: the veterinary perspective on eradication. Philosophical Transactions of the Roval Societv B 368:20120139.

Santos, N., V. Almeida, C. Gortázar, and M. Correia-Neves. 2015. Patterns of Mycobacterium tuberculosis-complex excretion and characterization of super-shedders in naturally-infected wild boar and red deer. Veterinary Research 46:129.

Storm, D., M. Samuel, R. Rolley, P. Shelton, N. Keuler, B. Richards, and T. Van Deelen. 2013. Deer density and disease prevalence influence transmission of chronic wasting disease in white-tailed deer. Ecosphere 4:1-14.

Thom, M., M. McAulay, H. Vordermeier, D. Clifford, R. Hewinson, B. Villarreal-Ramos, and J. Hope. 2012. Duration of immunity against Mycobacterium bovis following neonatal vaccination with bacillus Calmette-Guérin Danish: significant protection against infection at 12, but not 24, months. Clinical and Vaccine Immunology 19:12541260 .

Uehlinger, F., A. Johnston, T. Bollinger, and C. Waldner. 2016. Systematic review of management strategies to control chronic wasting disease in wild deer populations in North America. BMC Veterinarv Research 12:173.

Vicente, J., J. Barasona, P. Acevedo, J. Ruiz-Fons, M. Boadella, I. DíezDelgado, B. Beltran-Beck, and C. Gortázar. 2013. Temporal trend of tuberculosis in wild ungulates from Mediterranean Spain. Transboundary and Emerging Diseases 60:92-103.

Vicente, J., U. Höfle, J. Garrido, P. Acevedo, R. Juste, M. Barral, and C. Gortázar. 2007. Risk factors associated with the prevalence of tuberculosis-like lesions in fenced wild boar and red deer in south central Spain. Veterinary Research 38:451-464.

Wasserberg, G., E. Osnas, R. Rolley, and M. Samuel. 2009. Host culling as an adaptive management tool for chronic wasting disease in white-tailed deer: a modelling study. Journal of Applied Ecology 46: 457-466.

Woodroffe, R. 1999. Managing disease threats to wild mammals. Animal Conservation Forum 2:185-193.

Associate Editor: Benjamin M. Bolker Editor: Yannis Michalakis 